\title{
Polarization and isolation control for quantum cascade lasers in the mid- infrared
}

Sheng Wu, Andrei Deev

Sheng Wu, Andrei Deev, "Polarization and isolation control for quantum cascade lasers in the mid-infrared," Proc. SPIE 8993, Quantum Sensing and Nanophotonic Devices XI, 89930 (31 January 2014); doi:

$10.1117 / 12.2041086$

SPIE. Event: SPIE OPTO, 2014, San Francisco, California, United States 


\title{
Polarization and isolation control for Quantum Cascade Lasers in the Mid-Infrared
}

\author{
Sheng Wu, Andrei Deev \\ PEER Institute \\ 738 Arrow Grand Circle, Covina, CA 91722
}

\begin{abstract}
There have been limited choices of optical materials in the Mid-Infrared for polarization control and subsequent isolation. We show several combinations of existing materials and optics that could realize polarization control and isolation for quantum cascade lasers in the MIR. Improvements in signal to noise ratio in MIR laser spectroscopy, as well as saturated absorption spectroscopy utilizing the isolation achieved, will be discussed.
\end{abstract}

\section{KEY WORD LIST}

quantum cascade laser; laser spectroscopy, polarization and isolation in the Mid-IR, Hollow Waveguide

\section{INTRODUCTION}

Control of polarization and isolation of the laser from feedback are of vital importance for many applications of lasers, especially in spectroscopy and communication. As a high gain laser source, Quantum Cascade lasers are especially susceptible to external feedback[1, 2]. However, currently there are very limited number of optical materials that are suitable for the control of polarization and consequently isolation in the mid-Infrared (MIR). Therefore, polarization control and isolation are quite challenging in the mid-Infrared.

We discuss the techniques we used in the MIR spectroscopy with quantum cascade lasers, and realize the control of polarization and isolation of the laser from feedback. Waveplates made of $\mathrm{MgF}_{2}$ and Rutile will be discussed. We also compare the different degree of isolation achieved with different polarizors, i.e. Rutile, Holographic Wire Grid, and nanoparticle linear film polarizors.

We then show that we could use the isolation to improve the signal to noise ratios of our Hollow Waveguide (HWG) based spectroscopy platform. We also demonstrate saturated absorption spectroscopy inside HWG with the isolation realized by the waveplate and polarizors.

Quantum Sensing and Nanophotonic Devices XI, edited by Manijeh Razeghi, Eric Tournié, Gail J. Brown, Proc. of SPIE Vol. 8993, 89930U · (c) 2014 SPIE · CCC code: 0277-786X/14/\$18 · doi: 10.1117/12.2041086 


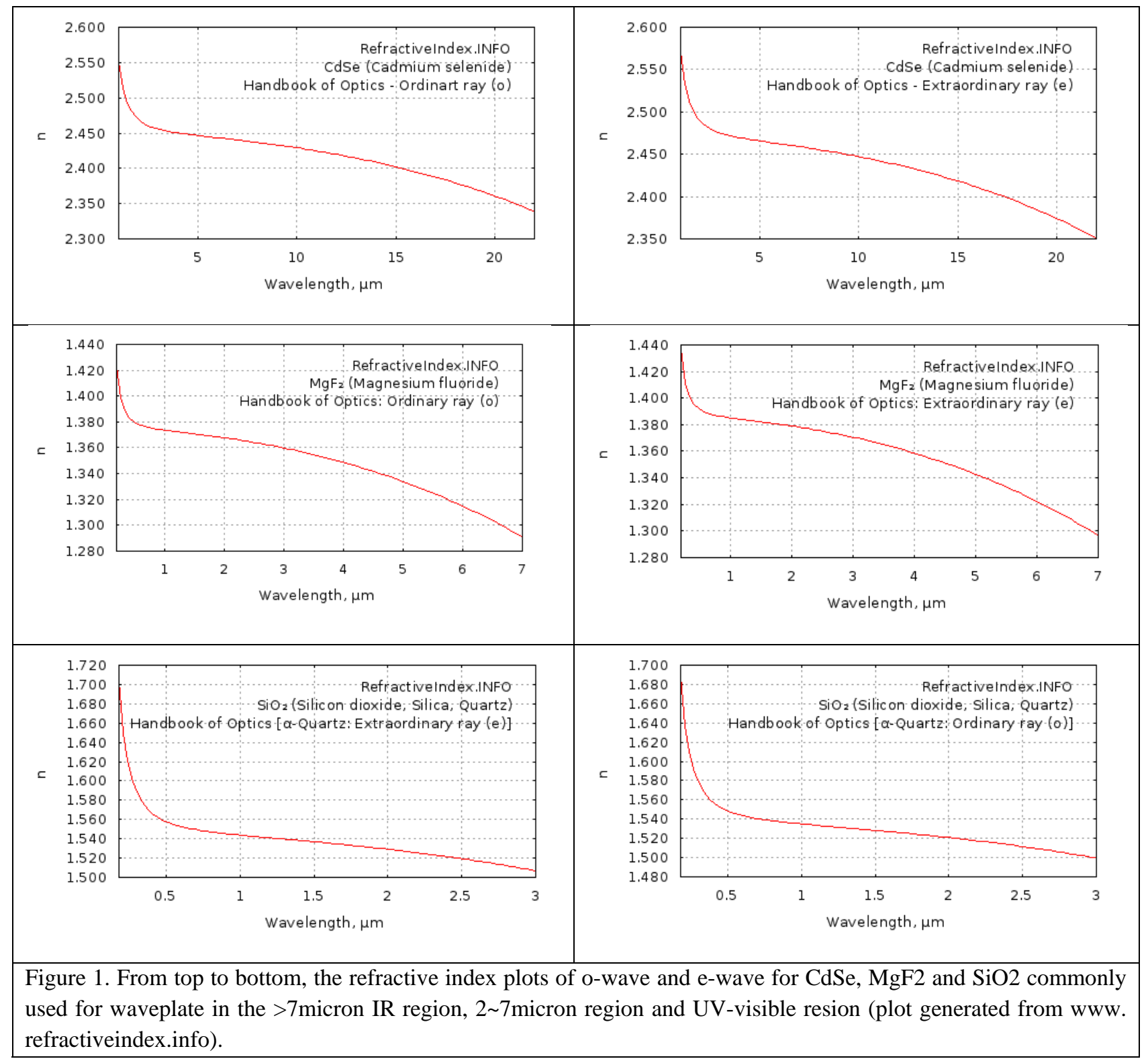

\section{EXPERIMENT}

\section{Waveplate and opto-magnetic rotator design for the Mid-Infrared}

The optical materials in the Mid-Infrared suffer much inferior transmission property when compared to visible and near infrared (NIR). The situation is exacerbated in the $>6$ micron range, where the birefringent crystals' transmission and other optical qualities could not be compared to the crystals in the visible or NIR. The longer wavelength in the Mid-IR usually requires thicker optical material to realize a waveplate, although this is beneficial for designing low order waveplate, the maximum thickness will be limited by the optical material's absorption loss. The appropriate materials for the Mid-IR region of $<7$ micron is usually MgF2; while for Mid-IR region $>7$ microns, CdSe and CdGa2S4 are often used, although the high refractive index and poor transmission means AR coating is necessary and thickness should be minimized.

We used the formula below to design a quarter waveplate for 4,500nm using MgF2 crystal, 


$$
\mathrm{L} \bullet \Delta \mathrm{n}_{\mathrm{eff}}=\lambda / 4, \Delta \mathrm{n}_{\mathrm{eff}}=\left(\mathrm{n}_{\mathrm{e}}-\mathrm{n}_{\mathrm{o}}\right)
$$

We arrived at $\mathrm{L}=115$ micron, for $\lambda=4,500 \mathrm{~nm}, \mathrm{n}_{\mathrm{e}}=1.351328$, and $\mathrm{n}_{\mathrm{o}}=1.341922$ for $\mathrm{MgF}_{2}$. Because MgF2 has very low refractive index, AR coating is almost unnecessary. However, we use the crystal at an off-axis angle of about $8^{\circ}$ to minimize feedback, and this is facilitated by adding a axis of rotation in the waveplate mount and it is quite critical to accurately adjust it during waveplate setup.

For magneto-optic effect based optical isolator in the Mid-IR, the choice of magneto-optic crystals are simply unavailable. So, we have to rely on simple but crude double passing quarter waveplate plus polarizer design to cut down undepolarized feedback. The depolarized feedback usually could not be filtered and therefore, we have to rely on the QC laser's low response to orthogonal polarization feedback to realize the isolation.

\section{Polarizer design for the Mid-Infrared}

Wire-grid polarizer (WGP) is almost the only choice for the Mid-Infrared. However, WGP suffers from poor transmission $\sim 75 \%$, and relatively low extinction ratio ( 150:1). Relatively new variant of WGP nanoparticle linear film polarizers and Mid-IR Wire Grid polarizers (available from Thorlabs.com) claim much better transmission and higher extinction ratio (>1,000:1). Tutile has large birefringence but limited transmission in the Mid-IR, and has been the only birefringent crystal that could realize high optical extinction ratio, e.g. 10^5:1 or higher.

\section{RESULTS}
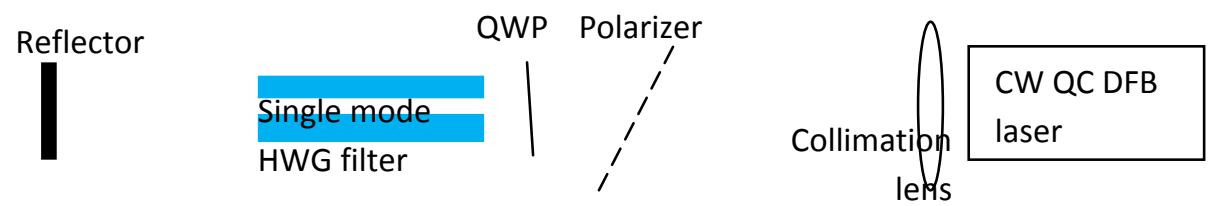

Figure 2 above shows the optical isolation that we tested for our hollow waveguide spectrometer. We put the Quarter waveplate (QWP) and polarizer after the collimation lens, and then a single mode Hollow Waveguide (HWG) filter (optional) is tested in before the Reflector. We align the Reflector so that light is directly reflected back into the laser to create feedback. This is illustrated in figure 3 a.

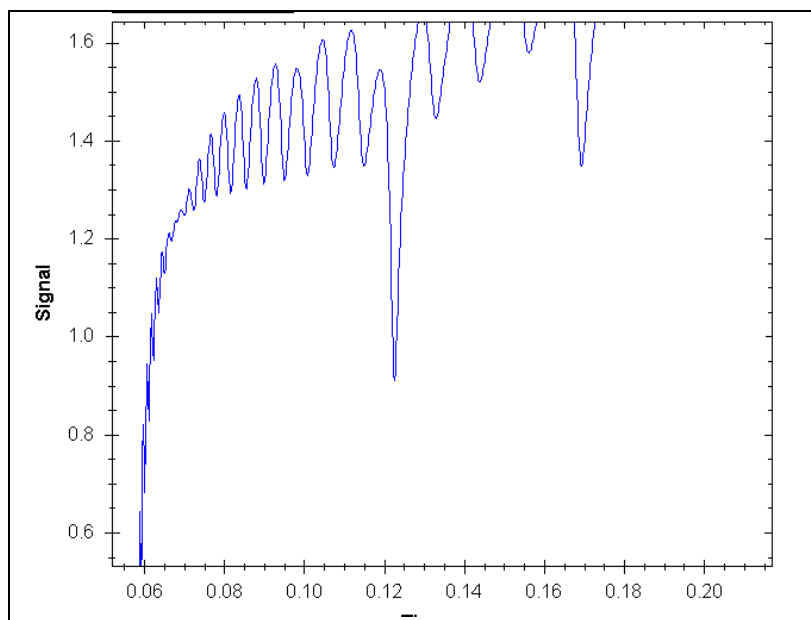

Figure 3a. Strong feedback after Reflector is aligned to created direct reflection back to QC laser. The two dips at $0.125 \mathrm{msec}$ and $0.17 \mathrm{msec}$ are $\mathrm{CO} 2$ absorption features.

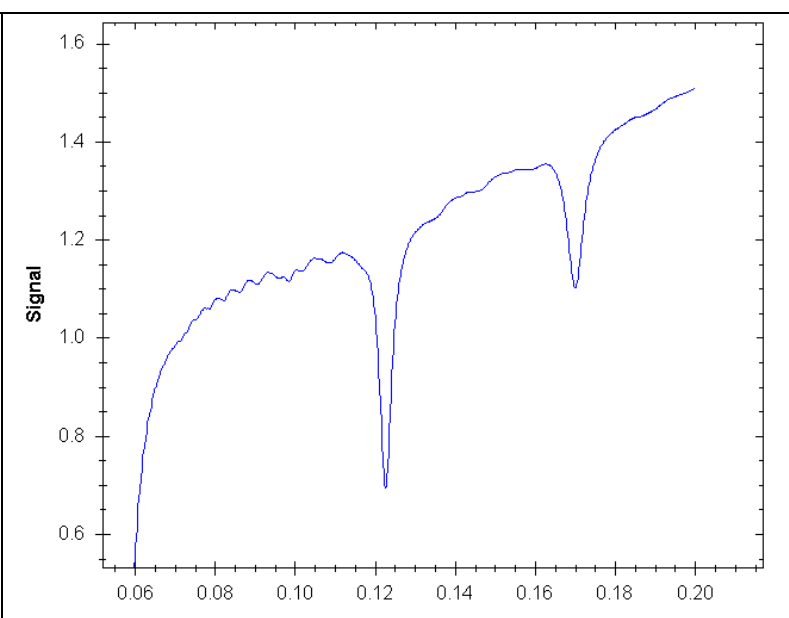

Figure 3b. Feedback reduced after QWP optimized and polarizer inserted. A third small feature at $0.09 \mathrm{msec}$ is now quite obvious. 
From figure 3, we could see the amplitude of feedback is cut down from $0.3 \mathrm{~V}$ to $0.03 \mathrm{~V}$, i.e. a 10 fold difference in feedback induced oscillation. With the HWG filter in place, another reduction up to 10 folds could be realized. We attribute this additional reduction due to the fact that HWG filter limited the scattering of the Reflector which could be depolarized and could not be filtered by the simple quarter waveplate plus polarizer isolator scheme.

Figure 4 below shows our Infrared Isotope Ratio (IR2) spectrometer which utilize such an isolation scheme. The optical isolation is necessary even for the single pass HWG sample cell due to scattering at the windows, non-ideal mode reflections inside the HWG, and even detectors at the end of the sample cell. With optical isolation, our IR2 spectrometer could achieve $<10^{-5} / \mathrm{Hz}^{1 / 2}$ resolution in absorption. We expect that with better optical arrangement, e.g. larger angle of incidence for optical windows/wedges at the HWG cell and detectors could further reduce the feedback and achieve $<10^{-6} / \mathrm{Hz}^{1 / 2}$ resolution.
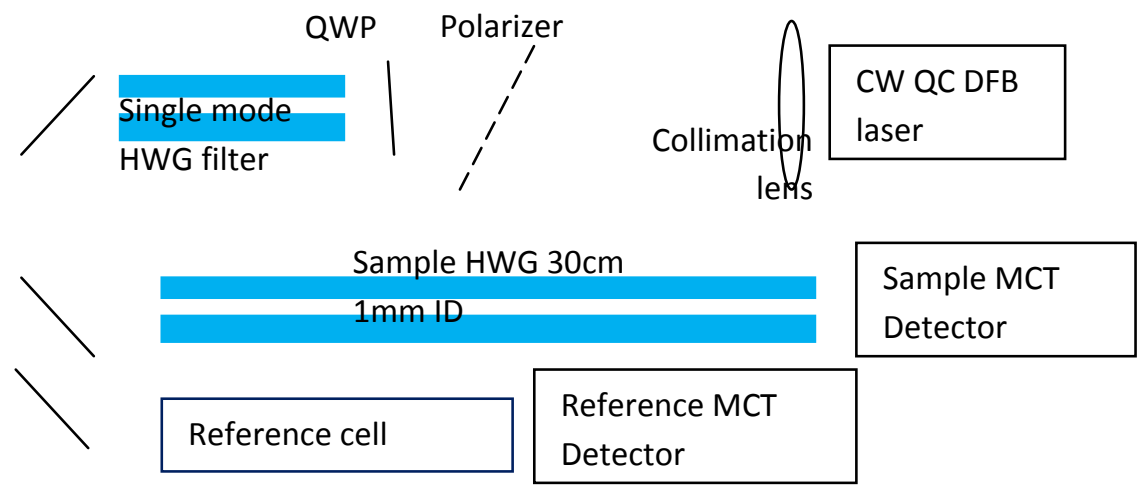

Figure 4. Optical train layout of our IR2 (Infrared Isotope Ratio) analyzer module

\section{DISCUSSIONS}

The effectiveness of the simple yet crude isolator consists of a quarter waveplate and polarizer is demonstrated. The isolation of $20 \mathrm{~dB}$ is demonstrated. Additional isolation might be difficult because the lack of isolation to depolarized feedback.

\section{CONCLUSION}

Here, we first give an overview of optical material for waveplate and polarizers, and design paradigm of waveplate in the Mid-IR. We then apply the quarter waveplate along with a grid polarizer in the QC laser absorption spectroscopy platform, where we demonstrated over $20 \mathrm{~dB}$ isolation of back reflected light. The isolation is necessary to achieve the high signal to noise ratio high precision absorption spectroscopy in our Infrared Isotope Ratio spectrometer.

\section{ACKNOWLEDGEMENT}

We gratefully acknowledge the financial support from DOE-RPSEA project titled 'Novel Gas Isotope Interpretation Tools to Optimize Gas Shale Production’ (DE-AC26- 07NT42677).

\section{REFERENCES}

1. Nelson, D.D., McManus, J. B., Herndon, S. C., Shorter, J. H., Zahniser, M. S., Blaser, S., Hvozdara, L., Muller, A., Giovannini, M., Faist, J., Characterization of a near-room-temperature, continuous-wave quantum cascade laser for long-term, unattended monitoring of nitric oxide in the atmosphere. Optics Letters, 2006. 31(13): p. 2012-2014. 
2. Kelly, J.F., et al., A capillary absorption spectrometer for stable carbon isotope ratio ([sup 13]C/[sup 12]C) analysis in very small samples. Review of Scientific Instruments, 2012. 83(2): p. 023101-14.

Proc. of SPIE Vol. $899389930 U-5$

Downloaded From: https://www.spiedigitallibrary.org/conference-proceedings-of-spie on 7/11/2018 Terms of Use: https://www.spiedigitallibrary.org/terms-of-use 